\title{
Baseline Wander by ECG Finding
}

National Cancer Institute

\section{Source}

National Cancer Institute. Baseline Wander by ECG Finding. NCI Thesaurus. Code C114147.

An electrocardiographic recording in which the isoelectric line in one or more leads is vertically displaced resulting in low frequency upward and downward movements of the signal with varying amplitudes. (CDISC) 